\title{
REVIEW
}

\section{Hyperlenses and metalenses for far-field super-resolution imaging}

\author{
Dylan $\mathrm{Lu}^{1} \&$ Zhaowei Liu¹,2
}

The resolution of conventional optical lens systems is always hampered by the diffraction limit. Recent developments in artificial metamaterials provide new avenues to build hyperlenses and metalenses that are able to image beyond the diffraction limit. Hyperlenses project super-resolution information to the far field through a magnification mechanism, whereas metalenses not only super-resolve subwavelength details but also enable optical Fourier transforms. Recently, there have been numerous designs for hyperlenses and metalenses, bringing fresh theoretical and experimental advances, though future directions and challenges remain to be overcome.

$\mathrm{O}$ ptical microscopy has revolutionized many fields such as microelectronics, biology and medicine. However, the resolution of a conventional optical lens system is always limited by diffraction to about half the wavelength of light. This diffraction barrier arises from the fact that subwavelength information from an object is carried by high spatial-frequency evanescent waves, which only exist in the near field. To overcome this diffraction limit, pioneering work has been carried out, including near-field scanning microscopy ${ }^{1}$, as well as fluorescence-based imaging methods ${ }^{2,3}$. These scanning- or random sampling-based nonprojection techniques achieve super-resolving power by sacrificing imaging speed, making them uncompetitive for dynamic imaging.

Lens-based projection imaging remains the best option for high-speed microscopy. Immersion techniques have been proposed to enhance resolution, but they are limited by the low refractive indices of natural materials ${ }^{4}$. Emerging within the last decade, the fields of plasmonics and metamaterials provide solutions for engineering extraordinary material properties not found in nature, such as negative index of refraction ${ }^{5,6}$ or strongly anisotropic materials ${ }^{7,8}$. This has provided tremendous opportunities for novel lens designs with unprecedented resolution 9 Initiated by Pendry's seminal concept of the perfect lens ${ }^{10}$, a number of superlenses were demonstrated with resolving powers beyond the diffraction limit ${ }^{11-17}$. The optical superlens first achieved sub-diffraction-limited resolution by enhancing evanescent waves through a slab of silver ${ }^{11}$. As the evanescent-field enhancement is enabled by surface plasmon excitation, the subwavelength image is typically limited to the near field of the metal slab. However, the hyperlens-a metamaterial-based lens-can send super-resolution images into the far field by introducing a magnification mechanism. This has been considered as one of the most promising candidates for practical applications since its first demonstration in 2007 (refs 13,14). Recently, various other metamaterial-based lenses, that is, metalenses, have also been developed with both

\footnotetext{
${ }^{1}$ Department of Electrical and Computer Engineering, University of California, San Diego, 9500 Gilman Drive, La Jolla, California 92093 , USA. ${ }^{2}$ Materials Science and Engineering, University of California, San Diego, 9500 Gilman Drive, La Jolla, California 92093, USA. Correspondence should be addressed to Z.L. (email: zhaowei@ece.ucsd.edu).
} 
super-resolving power and Fourier transform function, making them more like conventional lenses but with superior capabilities $^{18-21}$.

In this review, we will describe the underlying physics of the hyperlens and metalens, their design principles, recent advances, major limitations and practical challenges, as well as suggestions for future development. The extension of the hyperlens concept from electromagnetic waves to acoustic waves is also included. In addition, we will discuss some of the other super-resolution techniques, such as near-field scattering and the time-reversal technique.

\section{Physics of the hyperlens}

Light emission or scattering from objects comprises both propagating and evanescent components, corresponding to low and high wave-vectors, respectively. The propagating waves carrying large-feature information reach the far field, whereas the evanescent waves containing the detailed information are non-propagating in a normal material environment, and are thus confined to the near field. Diffraction-limited imaging originates from the fact that the deep subwavelength information carried by the evanescent waves cannot reach the far field and so does not contribute to the formation of the final image.

The realization of the hyperlens for far-field super-resolution imaging relies on two basic requirements: a material that supports wave propagation with high wave-vectors; and a magnification mechanism, that is, conversion of high wave-vector waves to low wave-vector waves so that the super-resolution information can be sent to the far field. Anisotropic plasmonic metamaterials provide one of the most practical options to fulfil the material requirement, for which there is no need to deal with the design of permeabilities but only permittivities along different directions; thus, the overall material loss is significantly reduced $^{8,22}$. The simplest anisotropic plasmonic metamaterials can be constructed by the deposition of alternating metal/dielectric multilayers (Box 1). When the layer thickness is much smaller than the probing wavelength, an effective-medium approximation is commonly used to describe the permittivities along different directions, that is, $\varepsilon_{x}, \varepsilon_{y}$ and $\varepsilon_{z}$ (ref. 23). Although the term 'hyperlens' was applied to metamaterials with a hyperbolic dispersion relationship, that is, $\varepsilon_{x}=\varepsilon_{y}$ and $\varepsilon_{z}$ taking different signs ${ }^{15}$, elliptically dispersive metamaterials can also be used to build a hyperlens as long as the coverage for lateral wave-vectors is large enough ${ }^{24}$. The magnification mechanism, that is, the wave-vector compression, is obtained by bending the flat layers into co-centrically curved layers 9,15 , which can be explained through transformation optics ${ }^{25}$. Owing to the conservation of angular momentum, the tangential wave-vectors are progressively compressed as the waves travel along the radial direction. A magnified image carried by low wave-vectors will ultimately be formed at the outer boundary of the hyperlens before propagating into the far field, supported by the surrounding environment. The magnification at the output surface is given simply by the ratio of the radii at the two boundaries.

Practical configurations of the hyperlens were proposed by different groups, including a cylindrical geometry ${ }^{15,25,26}$, tapered metallic wire arrays ${ }^{27-30}$ or uniquely designed material dispersions ${ }^{31-34}$. To circumvent the problem of absorption in real materials and thereby avoid image distortions in the far field, proper configurational design must ensure that all rays originating from the object plane travel equal path lengths through the metamaterial. Scattering at surfaces of the hyperlens, however, has proven detrimental for real imaging applications. Design rules have been proposed to impedance match the hyperlens metamaterial with both inner and outer boundaries
BOX 1 Hyperlens and anisotropic metamaterials.

Coarse and fine information from objects, corresponding to low and high wave-vector components, is carried by propagating and evanescent waves, respectively. Only propagating waves can reach the far field to be collected by a lens system, leading to the so-called 'diffraction limit'. Unlike isotropic media with a spherical dispersion, the dispersion properties for multilayer metamaterials can be designed to have $\varepsilon_{x}^{\prime}>0$ and $\varepsilon_{z}^{\prime}<0$ for hyperbolic dispersions or $\varepsilon_{z}^{\prime} \gg \varepsilon_{x}^{\prime}>0$ for eccentric elliptic dispersions, where $\varepsilon_{x}^{\prime}$ and $\varepsilon_{z}^{\prime}$ represent the real part of the permittivity along $x$ and $z$ directions (top left). Anisotropic metamaterials support the propagation of high wave-vector waves due to their unbounded wave-vector values or very large wave-vector cutoff. By using different material combinations, the working wavelength for multilayered metamaterials can be tuned across a broad band of wavelengths. Metallic nanowire-based metamaterials can also be used but cause shifts towards much longer operational wavelengths. The wavelength range for either hyperbolic or elliptic dispersion is determined by not only the constituents' permittivity but also their filling ratios. Anisotropic multilayer metamaterials in a curved geometry result in hyperlenses with corresponding dispersions represented in cylindrical coordinates (top right). For a subwavelength object placed at the inner boundary, wave propagation along the radial direction gradually compresses its tangential wave-vectors, resulting in a magnified image at the outer boundary. The magnified image, once larger than the diffraction limit, will be resolved in the far field. The bottom row shows that two sub-diffraction-limited line sources separated by a distance of $\sim 80 \mathrm{~nm}$ can be clearly resolved by using either eccentric elliptic (left) or hyperbolic (middle) metamaterials, but not by air alone (right). In the simulation, the radii of the hyperlens of either dispersion at the inner and outer boundaries are $\rho_{\text {inner }}=240 \mathrm{~nm}$ and $\rho_{\text {outer }}=1,200 \mathrm{~nm}$, respectively. The permittivity for metal and dielectric in the hyperbolic (elliptic) hyperlens is $-2.3-0.3 \mathrm{i}(-2.3-0.3 \mathrm{i})$ and $2.7(2.25)$, respectively. The filling ratio of metal in both hyperlenses is $50 \%$.
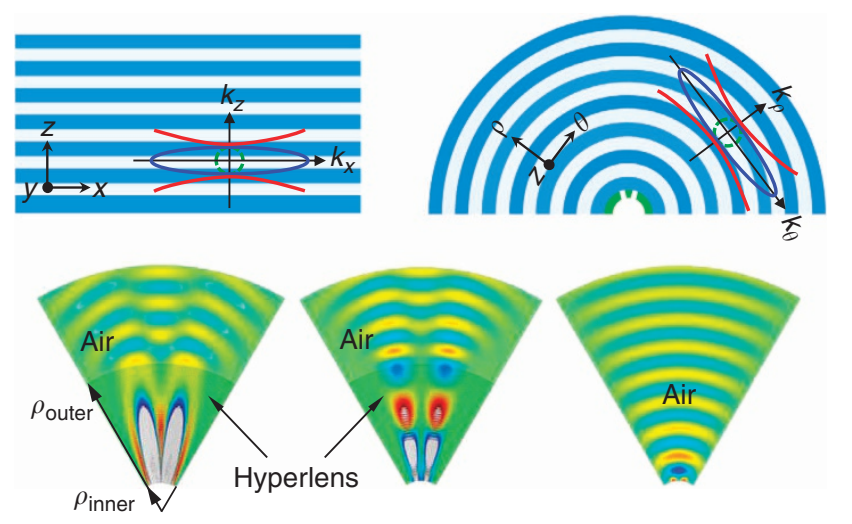

using radius-dependent magnetic permeability ${ }^{35}$. Comparable performance through the reduction of surface reflections can be achieved in non-magnetic metamaterials by impedance matching at one of the hyperlens surfaces instead of both. This trade-off has been confirmed by simulations and can be practically implemented $^{35,36}$. Owing to its use of curved surfaces, the cylindrical hyperlens design may not be convenient for bio-imaging. Design of the planar hyperlens has been shown to be theoretically feasible via specific material dispersion based on transformation optics ${ }^{31,32,37}$. In such designs, the metamaterial properties are designed to bend light rays from subwavelength features in such a way as to form a resolvable image on a flat output plane.

Experimental hyperlens demonstration and recent advances Theoretical predictions about hyperlenses, using anisotropic media composed of curved multilayer stacks ${ }^{15,26}$, have inspired 
a

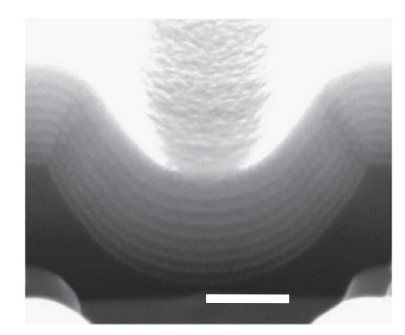

d

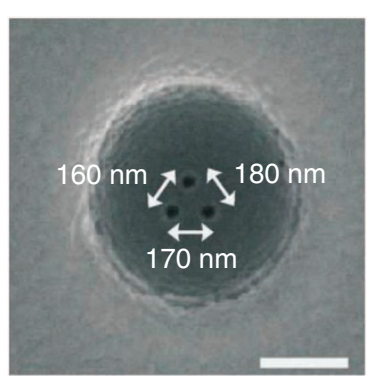

b
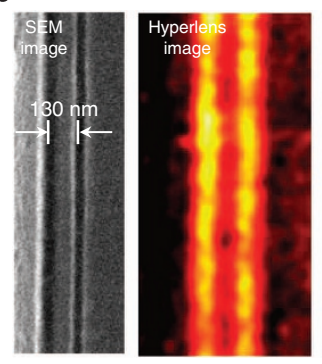

e

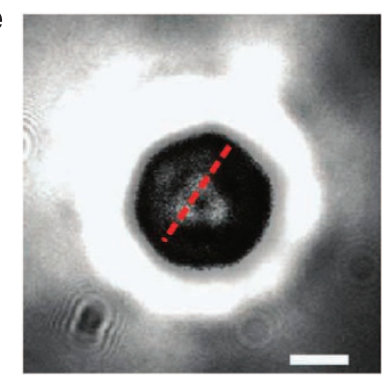

c
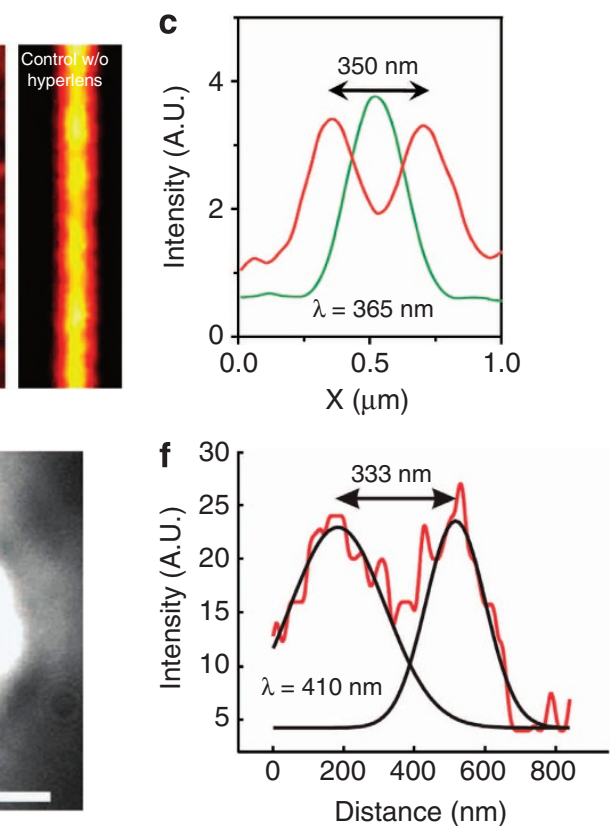

Figure 1 | Experimental demonstration of the optical hyperlens. (a) Scanning electron microscope (SEM) image of the cross section of a cylindrical hyperlens structure with $16 \mathrm{Ag}$ (bright) and $\mathrm{Al}_{2} \mathrm{O}_{3}$ (dark) layers. Reproduced with permission from ref. 38. The thick bright layer at the top is chromium. All scale bars shown in the figure are $500 \mathrm{~nm}$. (b) Hyperlens imaging of a pair of line objects with a line width of $35 \mathrm{~nm}$ and a centre-to-centre distance of $130 \mathrm{~nm}$. From left to right, SEM image of the line-pair object, magnified hyperlens image, and the resulting diffraction-limited image in a control experiment. (c) The cross-section profile for the hyperlens image of the line-pair object (red) and for a diffraction-limited image obtained without the hyperlens (green). A.U., arbitrary units. (b,c) From ref. 13 (reproduced with permission from AAAS). (d) SEM image of three dots positioned triangularly with gaps of 180 , 170 and $160 \mathrm{~nm}$ on a spherical hyperlens comprises nine pairs of $\mathrm{Ag}$ and $\mathrm{Ti}_{3} \mathrm{O}_{5}$ layers. (e,f) Image of the object after being magnified and its cross section along the red dashed line. The black curves in (f) correspond to the cross-sectional analysis for identifying the separation of the two apertures measured to be $333 \mathrm{~nm}$. (d-f), From ref. 17.

experimental investigations into the hyperlens concept. The first one-dimensional (1D) optical hyperlens was demonstrated at ultraviolet frequencies by conformal deposition of $\mathrm{Ag}$ and $\mathrm{Al}_{2} \mathrm{O}_{3}$ multilayer films on a cylindrical quartz cavity (Fig. 1a) $)^{13,38}$. A subdiffraction-limited object with a 130-nm centre-to-centre distance was observed directly in the far field (Fig. 1b,c). The magnified hyperlens image shows the object clearly resolved, as compared with a diffraction-limited image in a control experiment. So far, hyperlens experiments have achieved $125 \mathrm{~nm}$-pitch resolution $(\lambda / 2.92)$ working at a wavelength of $365 \mathrm{~nm}$ (ref. 38), although some simulations provided designs with much higher resolution around $\lambda / 9$ (ref. 39). A different approach to realizing cylindrical magnifying superlenses uses surface plasmon waves confined on a metal surface with a concentric polymer grating ${ }^{14}$. The magnified images of the surface waves after scattering by the surface roughness were collected by a microscope.

The cylindrical hyperlens only provides 1D resolution improvement, which limits its use in practical applications. A spherical hyperlens with two-dimensional (2D) superresolution capability is far more desirable, and has recently been experimentally demonstrated for the visible spectral region ${ }^{17}$. This $2 \mathrm{D}$ hyperlens was created by deposition of alternating layers of $\mathrm{Ag}$ and $\mathrm{Ti}_{3} \mathrm{O}_{5}$ thin films in a hemispherical geometry designed with a hyperbolic dispersion that supports the propagation of very high spatial-frequency waves at the same phase speed. An object with spacing down to $160 \mathrm{~nm}$ in a triangular configuration on the spherical hyperlens (Fig. 1d) is clearly resolved in the far field (Fig. 1e), beyond the diffraction limit, $205 \mathrm{~nm}$, at a wavelength of $410 \mathrm{~nm}$. The corresponding separation of the image is measured to be $333 \mathrm{~nm}$ in the cross-sectional analysis shown in Fig. 1f, resulting in an averaged magnification factor of 2.08 .

These proof-of-concept experiments have produced hyperlenses with hyperbolic dispersions working at ultraviolet and visible wavelengths. With appropriate material combinations, a broad working range across the visible or near-infrared becomes attainable for both hyperbolic dispersions and highly elliptical dispersions ${ }^{36}$. The resolution of hyperlenses is essentially determined by the geometry, material loss and also the film quality. In addition to increasing the magnification with a higher ratio of the outer-to-inner radii, the resolution could be further improved through the choice of appropriate filling ratios of metal and dielectric components because, as the dispersion approaches flatness, it results in the transport of even higher spatialfrequency information ${ }^{36}$. Although the intrinsic metal loss is unavoidable, the hyperlens can be optimized by increasing the amount of the dielectric component or working at longer wavelengths. A higher optical transmission can also be realized with resonant structures ${ }^{40,41}$. Moreover, instead of conformal film deposition, fabrication based on rolled-up technology provides an alternative method for controlling film conformality and roughness ${ }^{41-44}$.

Another super-resolution scheme based on metallic wire arrays was proposed to attain a wide range of working wavelengths over a long transport distance ${ }^{27}$. Experimental demonstrations, however, were limited to subwavelength imaging in the near field using metallic wires grown in dielectric templates ${ }^{45,46}$. The tapered nanorod array with a magnification mechanism for far-field imaging still suffers critical challenges in achieving accurate growth at tapered angles and introducing gaps between 
The essence of a lens is to modify the phase of an incident plane wave to form a focus. There are generally three methods to introduce a phase compensation mechanism into a lens design: shaping the surface of a piece of homogenous material, such as polishing a piece of glass to have a convex surface; use of diffractive structures to engineer the wavefront, as is done with the Fresnel zone plate; using material inhomogeneity to modify the phase change in space with, for instance, a GRIN lens. The metalens applies similar phase compensation mechanisms to metamaterials to bring a plane-wave to a focus. To achieve practical sub-diffraction-limited resolution, the metamaterials have to satisfy the same material requirements as those for a hyperlens. As high spatial-frequency waves inside a metamaterial cannot transmit to air due to total internal reflection, a bidirectional coupling mechanism is needed to convert waves from high spatial frequency in the metamaterial to low spatial frequency in air, or vice versa. Analogous to conventional lenses, various coupling schemes can fulfil this task, including metamaterials with shaped interfaces (left), a planar plasmonic metal-insulator-metal waveguide coupler (middle) and GRIN metamaterials (right). When the focal length is defined, the imaging magnification is obtained by placing an object at an appropriate distance from the metalens, which is similar to the case of a conventional lens.
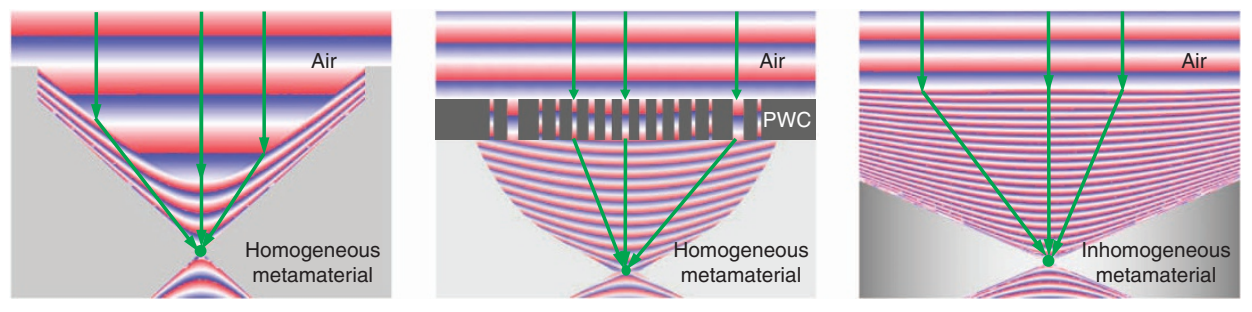

the arrays ${ }^{47}$. With increased efforts towards the development of growth technologies ${ }^{48-50}$, experimental demonstrations of the proposed scheme for subwavelength colour imaging could become a reality in the near future.

\section{Principles of the metalens}

The hyperlens discussed so far, although capable of superresolution for far-field detection, lacks the ability to focus plane waves. For conventional optical lenses, refraction and focusing of light rely on curved interfaces, fundamental for Fourier transforms and imaging. However, hyperlenses cannot realize the Fourier transform function due to their lack of a phase compensation mechanism. In this and the following sections, we will introduce the metalens: a metamaterial-based lens that provides not only the advantages of super-resolving capabilities but also the Fourier transform function, making it an exceptional combination of super-resolution and desirable functions of conventional lenses.

A metalens is created by combining a metamaterial slab and a phase compensation mechanism to bring a plane wave to a focus (Box 2). Anisotropic metamaterials with either a hyperbolic or eccentric elliptic dispersion are essential for supporting the propagation of super-resolution information carried by high wave-vectors. The hyperlens and the metalens thus share the same material requirement, with practical realizations including multilayers $^{13}$ and nanowire metamaterials ${ }^{22}$. On the other hand, as high wave-vector waves are totally reflected at the metamaterial-air interface, a bidirectional coupler is needed to convert waves from high wave-vectors in the metamaterial to low wave-vectors in air, and vice versa. To make a focusing lens, this coupler must also function as a phase compensation mechanism, in contrast with the far-field superlens that functions as an evanescent-to-propagating convertor through the use of a grating $^{16}$.

Various types of phase compensation mechanism have been proposed for focusing plane waves in metalenses ${ }^{18-20}$. The underlying design principle is to satisfy the phase matching condition to constructively bring plane waves from air into deep subwavelength focus inside the metamaterial. This can be accomplished by either geometric variations, such as plasmonic waveguide couplers (PWC) ${ }^{18,51-53}$ and shaped metamaterial-air interfaces ${ }^{19,54}$, or material refractive-index variations like gradient-index (GRIN) metamaterials ${ }^{20}$. For example, a PWC, composed of an array of specially designed non-periodic metalinsulator-metal waveguides, can turn non-flat wave-fronts at the metamaterial-PWC interface into flat ones at the PWC-air interface, thus matching the phase condition for realizing a deep subwavelength focus inside the metamaterial slab ${ }^{18,51}$. Instead of relying on metal-insulator-metal waveguides, a metamaterial with a hyperbolic dispersion can also achieve low and high wavevector conversion and phase compensation after being shaped to a concave surface. In contrast, a convex surface is needed for metamaterials with an elliptic dispersion. The curvature of the metalens is determined by a lens maker's equation that is similar to the conventional one ${ }^{19}$. Using transformation optics ${ }^{55,56}$, such geometry variations can be transformed to refractive-index variations within the material space, resulting in a GRIN metalens. Analogous to a conventional GRIN lens ${ }^{57}$, the designed permittivity profiles for a GRIN metalens gradually bring plane waves to a super-resolution focus inside the metamaterial $^{20,58}$.

\section{Plane-wave focusing for optical Fourier transforms}

One demonstration of the metalens was first proposed by utilizing a planar PWC at ultraviolet wavelengths ${ }^{18}$. Based on a silver PWC, the metalens with an elliptic dispersion achieved a deep subwavelength focal spot with a full width at half maximum of $59 \mathrm{~nm}(\sim \lambda / 6.2)$ in the metamaterial ${ }^{18}$. Compared with the diffraction-limited focal spot in air, about threefold resolution improvement was demonstrated. A similar improvement for a hyperbolic metalens based on a planar aluminium PWC is also possible $^{18}$. Under the same design principle, an alternative PWC satisfies the phase condition by tuning the waveguide height instead of width. Improved resolution $(\sim \lambda / 8.6)$ was verified at longer wavelengths, due to smaller material loss ${ }^{51}$.

Although the PWC-based metalens is capable of exceptional imaging resolution, it may demand a sophisticated fabrication process. As a metamaterial counterpart to the solid immersion lens ${ }^{4,59}$, metalenses formed by shaping metamaterial surfaces have recently been proposed for super-resolution imaging with the Fourier transform function ${ }^{19}$. Using a convex geometry, an elliptic metamaterial made of multilayers of silver and gallium phosphide can satisfy the phase compensation condition for subwavelength focusing. A deep subwavelength focal spot with a full width at half maximum of $70 \mathrm{~nm}(\sim \lambda / 9)$ was formed at a focal length of $3 \mu \mathrm{m}$ for normal plane-wave incidence at a red 


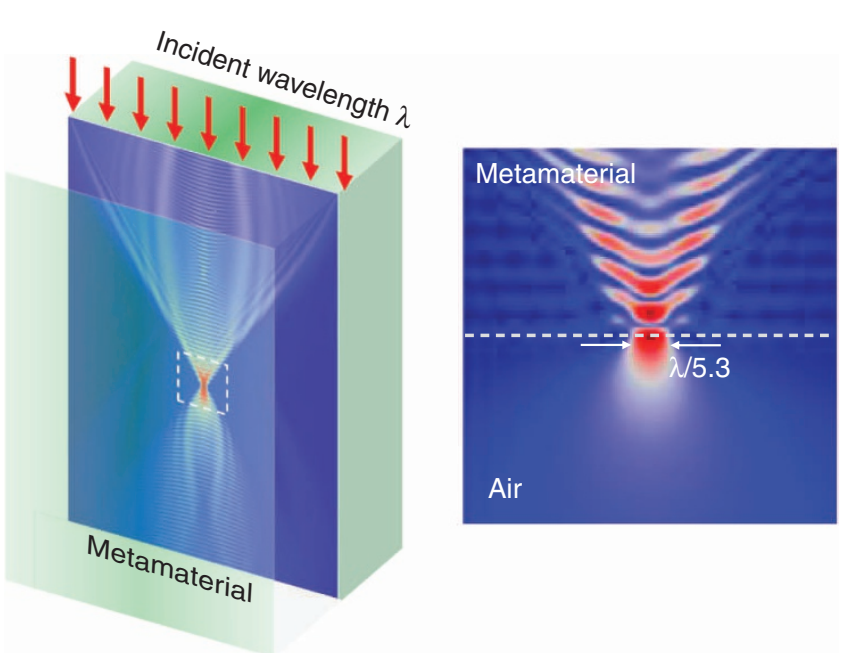

Figure 2 | Numerical demonstration of a GRIN metalens. A hyperbolic GRIN metalens forms a sub-diffraction-limited internal focus inside the metamaterial. A full width at half maximum of $156 \mathrm{~nm}(\lambda / 5.3)$ is obtained at the illumination wavelength of $\lambda=830 \mathrm{~nm}$ when the lens is truncated at the focal plane, as shown on the right, which displays a zoom-in of the boxed region around the focus. Reproduced, with permission, from ref. 20.

Copyright 2011 by the American Physical Society.

wavelength of $633 \mathrm{~nm}$ (ref. 19). This metalens achieved an effective numerical aperture of 4.5 , which is much larger than that found in naturally occurring materials ${ }^{60,61}$. In addition, owing to negative refraction, a hyperbolic metamaterial must be made into a concave geometry to focus light, which is exceptionally different from the requirement for conventional lenses ${ }^{19}$.

Such geometry manipulation can alternatively be converted to spatially variant material property design. Inhomogeneous metamaterials have been utilized to focus light in the infrared ${ }^{58,62}$ or surface waves ${ }^{63}$. The GRIN metalens, with appropriately designed permittivity profiles, was first investigated for its superresolution imaging, as well as its Fourier transform capabilities at optical wavelengths ${ }^{20}$. The hyperbolic permittivity profile, enabled by the use of silver nanowires grown in an alumina template, gradually converges a normally incident wave to a subdiffraction-limited focus inside the metamaterial (Fig. 2). When the metalens is truncated at a plane close to the internal focus, the super-resolution focus on the truncated surface can be accessed externally (Fig. 2).

All the metalenses introduced above were built based on real material properties. In addition to material and coupling loss, non-monochromatic illumination may reduce the focusing performance, as the metamaterials are made of dispersive metal and dielectric composites. Nonetheless, they work fairly well in a relatively wide range of wavelengths, and can always be optimized for further resolution improvement by careful choice of material combinations for desired working wavelengths. Here, we mainly focused on super-resolution focusing in $1 \mathrm{D}$ at optical frequencies. Yet these principles can be easily extended to $2 \mathrm{D}$, as well as to other frequencies, such as the infrared, terahertz, or microwave regions. The same principles can also be applied to acoustics due to the nature of waves.

Extraordinary imaging properties of the hyperbolic metalens The metalens exhibits deep subwavelength plane-wave focusing with a defined focal length, which is in stark contrast to the hyperlens. The imaging properties of a lens, that is, the image characteristics of an object placed at different locations with respect to the lens focal length $f$, are well understood, being a
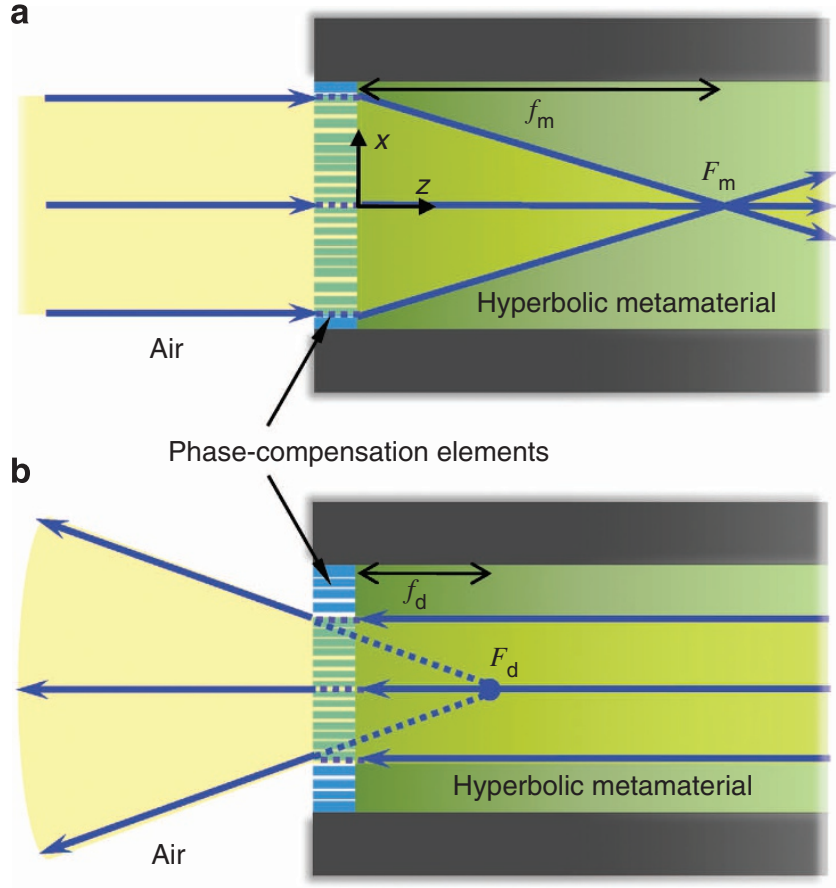

Figure 3 | Extraordinary focusing property of a Janus lens. (a) Plane waves from air converge through a phase-compensating element to a focus $\left(F_{\mathrm{m}}\right)$ in the metamaterial. (b) Plane waves from the metamaterial diverge in air, resulting in a virtual focus $\left(F_{d}\right)$ also in the metamaterial. The arrows in (a) and (b) are eye-guiding rays.

governed by the imaging equation $1 / s_{\mathrm{o}}+1 / s_{\mathrm{i}}=1 / f$, where $s_{\mathrm{o}}$ and $s_{\mathrm{i}}$ are the object and image distances to the lens, respectively. A metalens achieves subwavelength focusing by combining either hyperbolic or elliptically dispersive metamaterials with a phase compensation mechanism. Notice that the hyperbolic metalens has negative refraction at the metalens-air interface, leading to an opposite focal shift with respect to the tilted incident plane waves $^{18-20}$. Unprecedented imaging properties are expected from such lenses with abnormal Fourier transform properties ${ }^{21}$.

Consider a PWC-based hyperbolic metalens that comprises a PWC and a metamaterial slab with hyperbolic dispersion $\left(\varepsilon_{x}^{\prime}>0\right.$ and $\left.\varepsilon_{z}^{\prime}<0\right)$. A plane wave incident from the air side converges to the focus $F_{\mathrm{m}}$ at a focal length of $f_{\mathrm{m}}$ in the hyperbolic metamaterial side (Fig. 3a). Meanwhile, a plane wave from the metamaterial side diverges after passing through the metalens, resulting in a virtual focus at $F_{\mathrm{d}}$ at a focal length of $f_{\mathrm{d}}$ also in the metamaterial (Fig. 3b). In contrast to a converging lens with one focus on either side, this distinctive focusing behaviour makes the hyperbolic metalens into a 'Janus lens', having two different focusing behaviours in opposite directions.

For a Janus lens, the imaging behaviour is governed by a new metalens imaging equation ${ }^{21}$,

$$
\frac{1}{v_{\mathrm{d}}}+\frac{\varepsilon_{z}^{\prime} / \sqrt{\varepsilon_{x}^{\prime}}}{v_{\mathrm{m}}}=\frac{\varepsilon_{z}^{\prime} / \sqrt{\varepsilon_{x}^{\prime}}}{f_{\mathrm{m}}}
$$

or

$$
\frac{1}{v_{\mathrm{d}}}+\frac{\varepsilon_{z}^{\prime} / \sqrt{\varepsilon_{x}^{\prime}}}{v_{\mathrm{m}}}=\frac{1}{f_{\mathrm{d}}},
$$

where $v_{\mathrm{d}}$ and $v_{\mathrm{m}}$ are the image and object distances in air and metamaterial, respectively; the focal lengths satisfy $f_{\mathrm{d}}=f_{\mathrm{m}} \sqrt{\varepsilon_{x}^{\prime}} / \varepsilon_{z}^{\prime}$. In contrast to the conventional imaging equations, the material properties for a metalens also have an 


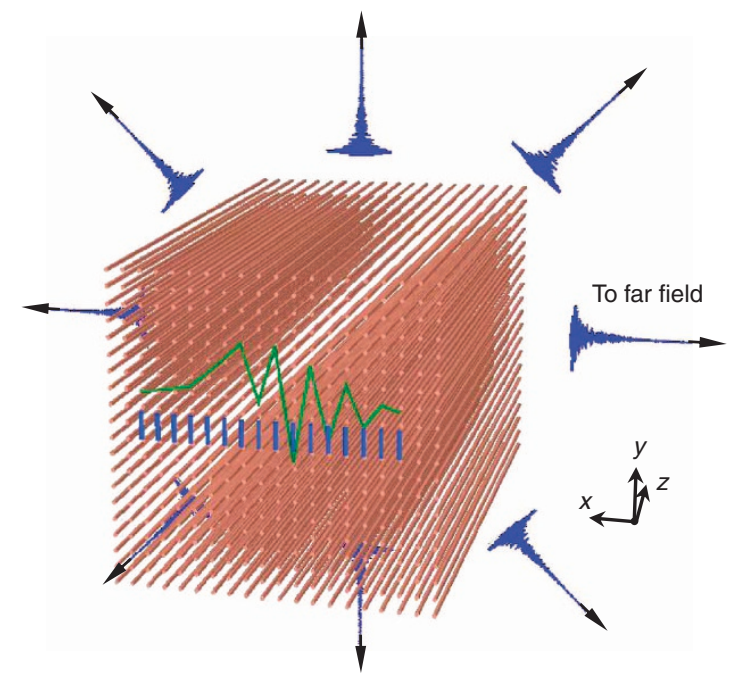

Figure 4 | Super-resolution based on time reversal. Schematic configuration of a resonant metalens made of an array of copper wires with a subwavelength period of about $\lambda / 80$ placed on the ground on top of a copper plate (the copper plate has been removed for clarity). A subwavelength object consisting of 16 small monopoles with a period of $\lambda / 80$ (blue line array) is placed between the ground plane and the metalens. The emission amplitude profile of the array is shown in green (data from ref. 66). Time-resolved signals scattered off the metalens propagate into the far field to be collected by far-field antennas in different directions.

important role in its imaging characteristics. Therefore, the Januslens imaging properties with respect to the focal lengths are completely different from those of a conventional glass lens ${ }^{64}$. For example, the Janus-lens image of an object in air is always minified, erect and real within the first focal length inside the metamaterial. In contrast, a glass lens image may be magnified, erect and imaginary if it is too close to the lens, as in the case for a magnifying lens.

When the metalens is truncated to a plane within $F_{\mathrm{m}}$, a magnified real image is formed in air for a subwavelength object placed on the truncated surface of the metamaterial ${ }^{21}$. Therefore, a super-resolution image for far-field detection becomes possible. Hyperbolic metalenses having this type of new imaging property, in addition to super-resolution, pave the way for novel optical devices and systems that could be extremely hard to achieve with conventional lenses.

\section{Super-resolution based on far-field inversion procedures}

Both hyperlenses and metalenses achieve super-resolution imaging with the use of anisotropic metamaterials that support the propagation of high spatial-frequency waves and a magnification mechanism for detection in the far field. Alternative approaches have been proposed for sub-diffraction-limited imaging utilizing inversion procedures with far-field detection, including time reversal, optical diffraction tomography (ODT) and compressed sensing ${ }^{65-70}$. The time-reversal technique was first introduced as an inversion method for electromagnetic waves and was experimentally demonstrated by subwavelength focusing and imaging of microwaves ${ }^{65,66,71-75}$. Evanescent waves that carry small details are converted into propagating waves by placing a subwavelength object made of monopoles in the near field of a cluster of strongly coupled resonators, referred to as a 'resonant metalens' (Fig. 4). In contrast to the metalens that uses a narrow band of frequencies, the resonant metalens takes advantage of the

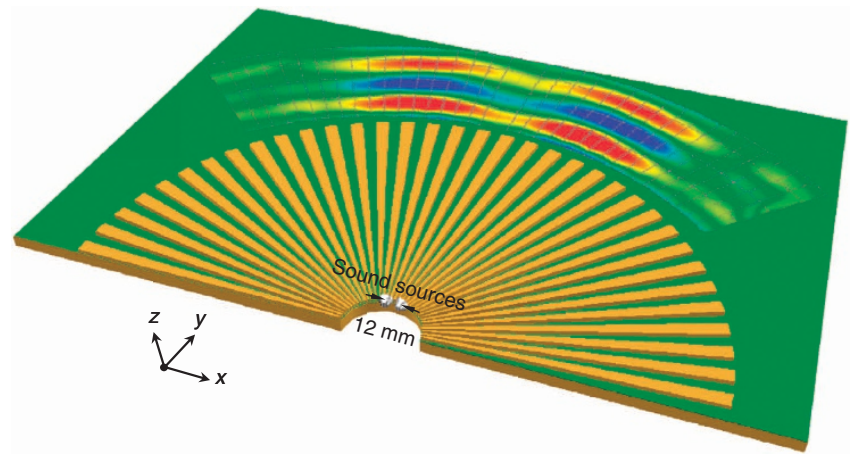

Figure 5 | Super-resolution imaging of acoustic waves. A 2D acoustic hyperlens made of 36 brass fins (running radially from a radius of 2.7$21.8 \mathrm{~cm}$ ) in air embedded on a brass substrate, spanning $180^{\circ}$ in the angular direction. The cover aluminium sheet has been removed to expose the microstructure for this view. The intensity profile in the green background represents experimental pressure measurements from ref. 87 in the propagation region that show a magnified image of a sub-diffraction-limited object consisting of two in-phase sound sources separated by $1.2 \mathrm{~cm}$ at $6.6 \mathrm{kHz}$. The pressure is measured at various points ranging from 0.4 to $9.9 \mathrm{~cm}$ away from the lens.

strong dispersive properties of the resonator structures. The broadband emission from the object generates a subwavelength profile at the input of the lens. Coupling between the source and the eigenmodes inside the resonant metalens efficiently scatters off near-field information, which is encoded in time and recorded by far-field antennas in different directions (Fig. 4). Using time reversal, the far-field detection is 'flipped' in time and reemitted from the same antenna locations. The time-reversed signal arrives at the lens and is recorded by the monopoles, now acting as receivers. Based on the reciprocity of the system, not only propagating waves but also evanescent waves can be recovered at the monopole location. An image with a sub-diffraction-limited resolution of $\lambda / 80$ has been reconstructed by cross-correlation between the received signals and the filters ${ }^{66,72}$. An extension to $2 \mathrm{D}$ imaging is possible by using a total internal reflection configuration $^{72}$. The resolution of such a resonant metalens is affected by the period of the wires, as well as the loss in metals, which cannot be compensated by time reversal. It may be possible to extend this proof-of-concept from the microwave range to the near-infrared or visible, although the details of resonator design and experimental methodology may be very different ${ }^{76,77}$.

In contrast to the inversion procedure based on temporal signal reversal, another approach, widely known as ODT or synthetic aperture digital holography, reconstructs the image using multiple measurements of both the amplitude and phase of the field diffracted by an unknown object at many incident angles ${ }^{78-80}$. Recently, it has been studied with the goal of breaking the diffraction limit in far-field optical imaging ${ }^{67,68,81-83}$. The resolution is improved by measurements at many incident angles that enlarge the accessible range of spatial frequencies. The permittivity or shape, intrinsic properties of the object, are then reconstructed numerically by well-developed inversion algorithms that iteratively minimize the discrepancy between the measured scatter field and that simulated via a forward solver $^{68}$. The resolution performance of ODT depends on various factors, including the number of incidences and observation points, covered angular range, inversion algorithms, and system noise level. Although a moderate resolution improvement has been demonstrated experimentally ${ }^{68}$, the imaging speed is limited by the need for multiple measurements. 


\section{Hyperlens for acoustic waves}

As in the case for electromagnetic waves, the resolution for conventional acoustic imaging is also limited by the wavelength of acoustic waves due to loss of spatial details carried by evanescent waves. The concept of anisotropic metamaterials has therefore been suggested for super-resolution imaging of acoustic waves for potential applications in sonar sensing, ultrasonic imaging and non-destructive material testing ${ }^{84-86}$. In 2009, a 2D acoustic hyperlens was experimentally demonstrated for deepsubwavelength imaging over a broadband frequency range and with low loss ${ }^{87}$. It consists of a non-resonant radially symmetric layered structure similar to the optical hyperlens, but with tapered metallic wires that gradually convert evanescent waves into propagating waves in the far field (Fig. 5). Two sound sources with a subwavelength separation of $1.2 \mathrm{~cm}$ were well resolved at the outer surface of the hyperlens when the frequency was swept from 4.2 to $7 \mathrm{kHz}$, corresponding to a $\lambda / 6.8$ to $\lambda / 4.1$ resolution. Owing to their longitudinal nature, the dispersion relation for acoustic waves is described by the effective mass density together with the effective bulk modulus ${ }^{88}$. Instead of using a hyperbolic dispersion, the acoustic hyperlens has a highly eccentric elliptic dispersion with positive effective mass density that supports a broadband working frequency and a large range of wave-vectors. With an elliptic rather than a hyperbolic dispersion, it avoids the use of resonating elements in constructing the metamaterial, resulting in a low-loss hyperlens with very large propagation distances and, thus, a very large magnification. This kind of anisotropic acoustic metamaterial was later utilized in the fabrication of a 3D holey-structured metamaterial for acoustic deep-subwavelength imaging ${ }^{89}$. A $2 \mathrm{D}$ object with a line width of $3.18 \mathrm{~mm}$ can be clearly resolved at the operating frequency of $2.18 \mathrm{kHz}$, demonstrating a subwavelength resolution of $\lambda / 50$ (ref. 89). This was achieved by the effective transmission of evanescent wave components carrying deep-subwavelength details to the output side through the Fabry-Pérot resonances excited inside the holey structure. Although the nearly perfect imaging was confined to the near field of the acoustic metamaterial, it points to a possibility for constructing a $3 \mathrm{D}$ hyperlens for far-field subwavelength imaging by perforating a hemispherical solid with tapered holes in the radial direction. In addition to deep subwavelength imaging, other applications of acoustic metamaterials for extreme manipulation of acoustic waves include focusing, cloaking and extraordinary transmission or absorption ${ }^{90-93}$.

Despite all the success achieved by acoustic metamaterials, other strategies such as time-reversal techniques ${ }^{94,95}$ have also been extended to overcome acoustic imaging limitations. Based on a sonic analogue to the resonant metalens proposed for electromagnetic waves ${ }^{65,66}$, one group recently proved by experiment that broadband sounds can be controlled and focused on a subwavelength scale by using acoustic resonators ${ }^{94}$. The demonstration of foci with a $\lambda / 25$ size for audible sound testifies to the generality of the time-reversal principle. Nevertheless, experimental proof of the capability of the time-reversal metalens for subwavelength imaging is still needed.

\section{Perspectives and outlook}

Over the past few years, the hyperlens, relying on anisotropic metamaterials with magnifying power, has been demonstrated to achieve sub-diffraction-limited resolution for far-field detection across the optical regime. On the other hand, the metalens has recently been proposed as a new member of the superlens family, having not only super-resolution power but also Fourier transform function due to the incorporation of a phase compensation mechanism, which makes it a more suitable candidate for integration with conventional optical systems. Although theoretical analysis and numerical simulations have proven its deep subwavelength focusing performance, as well as extraordinary imaging properties as a Janus lens, experimental demonstration of the metalens is necessary to prove its practical feasibility. In addition, there seem no physics limitations for the demonstration of the time-reversal imaging technique at optical frequencies, but necessary experimental technologies need to be developed before the technique can be effectively implemented. The possibilities for combining the time-reversal technique with hyperlenses or metalenses may yet become an interesting field worth exploring.

There are two major limitations to future applications of both hyperlenses and metalenses. First, material loss is an intrinsic property of metamaterials at optical wavelengths, limiting the ultimate resolution of the lens, as well as the signal transmission efficiency, especially when resonant elements are involved. To mitigate the loss problem, several approaches may provide solutions, such as compensating for loss in the metal by addition of a gain medium in dielectrics ${ }^{96}$, searching for better plasmonic materials among existing elements, band structure engineering or materials doping or alloying ${ }^{97,98}$. Second, the object has to be placed in the near field of the lens to make use of the evanescent waves that normally decay away from the object, although the image can be projected and detected in the far field. This is also true for super-resolution imaging based on time-reversal and ODT. Super-resolution is thus limited to surface imaging, which may encounter difficulties in extension to the third dimension. New imaging schemes may be needed to solve this issue.

Besides, current designs for both hyperlenses and metalenses have very limited field of view due to the limited physical size of the lens. Better designs, such as periodic array geometries with improved field of view to lens-diameter ratio, may solve the problem but it remains wide open to solutions. Image distortion and corresponding compensation methods are also important issues that need further investigations for practical applications of these lenses. In the case of hyperlenses, imaging at the back focal plane may result in a great reduction in distortions.

Despite existing limitations, super-resolution with hyperlenses and metalenses has already shown exciting potential applications in various fields of science and technology. The implementation of these lenses in conventional optical microscopes may extend the resolution to the nanometre scale for real-time observations, which would have a great impact on modern biological imaging. Further improvement is expected by combining hyperlenses or metalenses with other imaging techniques, such as dark-field microscopy for high contrast microscopy ${ }^{99}$. Super-resolution imaging and its reversed process for writing may find applications in optical lithography and ultrahigh-density optical data storage $^{39}$. The extension of the hyperlens and metalens concept to acoustic waves will also lead to super-resolution in applications such as sonar sensing and ultrasonic imaging.

\section{References}

1. Betzig, E. \& Trautman, J. K. Near-field optics: microscopy, spectroscopy, and surface modification beyond the diffraction limit. Science 257, 189-195 (1992).

2. Hell, S. W. Far-field optical nanoscopy. Science 316, 1153-1158 (2007).

3. Huang, B., Wang, W., Bates, M. \& Zhuang, X. Three-dimensional superresolution imaging by stochastic optical reconstruction microscopy. Science 319, 810-813 (2008).

4. Mansfield, S. M. \& Kino, G. S. Solid immersion microscope. Appl. Phys. Lett. 57, 2615-2616 (1990).

5. Soukoulis, C. M., Linden, S. \& Wegener, M. Negative refractive index at optical wavelengths. Science 315, 47-49 (2007).

6. Shelby, R. A., Smith, D. R. \& Schultz, S. Experimental verification of a negative index of refrraction. Science 292, 77-79 (2001).

7. Podolskiy, V. A. \& Narimanov, E. E. Strongly anisotropic waveguide as a nonmagnetic left-handed system. Phys. Rev. B 71, 201101(R) (2005). 
8. Podolskiy, V. A., Alekseyev, L. V. \& Narimanov, E. E. Strongly anisotropic media: the $\mathrm{THz}$ perspectives of left-handed materials. J. Mod. Opt. 52, 2343-2349 (2005).

9. Zhang, X. \& Liu, Z. Superlenses to overcome the diffraction limit. Nat. Mater. 7, 435-441 (2008).

10. Pendry, J. B. Negative refraction makes a perfect lens. Phys. Rev. Lett. 85, 3966-3969 (2000). First theoretical proposal for the perfect lens.

11. Fang, N., Lee, H., Sun, C. \& Zhang, X. Sub-diffraction-limited optical imaging with a silver superlens. Science 308, 534-537 (2005). First experimental demonstration of the superlens with a subwavelength resolution.

12. Taubner, T., Korobkin, D., Urzhumov, Y., Shvets, G. \& Hillenbrand, R. Near-field microscopy through a SiC superlens. Science 313, 1595 (2006).

13. Liu, Z., Lee, H., Xiong, Y., Sun, C. \& Zhang, X. Far-field optical hyperlens magnifying sub-diffraction-limited objects. Science 315, 1686-1701 (2007). First experimental demonstration of the 1D hyperlens with a subwavelength resolution at ultraviolet frequencies.

14. Smolyaninov, I. I., Hung, Y.-J. \& Davis, C. C. Magnifying superlens in the visible frequency range. Science 315, 1699-1701 (2007).

Experimental demonstration of the cylindrical magnifying superlens using surface plasmon waves at visible frequencies.

15. Jacob, Z., Alekseyev, L. V. \& Narimanov, E. Optical hyperlens: Far-field imaging beyond the diffraction limit. Opt. Express 14, 8247-8256 (2006)

16. Liu, Z. et al. Far-field optical superlens. Nano Lett. 7, 403-408 (2007).

17. Rho, J. et al. Spherical hyperlens for two-dimensional sub-diffractional imaging at visible frequencies. Nat. Commun. 1, 143 (2010).

Experimental demonstration of the $2 D$ hyperlens with a subwavelength resolution at visible frequencies.

18. Ma, C. B. \& Liu, Z. W. A super resolution metalens with phase compensation mechanism. Appl. Phys. Lett. 96, 183103 (2010).

Proposal and numerical demonstration of the metalens with deep subwavelength resolution by utilizing plasmonic waveguide coupler.

19. Ma, C. B. \& Liu, Z. W. Focusing light into deep subwavelength using metamaterial immersion lenses. Opt. Express 18, 4838-4844 (2010).

20. Ma, C. B., Escobar, M. A. \& Liu, Z. W. Extraordinary light focusing and Fourier transform properties of gradient-index metalenses. Phys. Rev. B 84, 195142 (2011)

21. Ma, C. \& Liu, Z. Breaking the imaging symmetry in negative refraction lenses. Opt. Express 20, 2581-2586 (2012).

Characterization and equational description of the extraordinary imaging properties of the hyperbolic metalens.

22. Yao, J. et al. Optical negative refraction in bulk metamaterials of nanowires. Science 321, 930 (2008).

23. Ramakrishna, S. A., Pendry, J. B., Wiltshire, M. C. K. \& Stewart, W. J. Imaging the near field. J. Mod. Opt. 50, 1419-1430 (2003).

24. Ma, C., Aguinaldo, R. \& Liu, Z. Advances in the hyperlens. Chin. Sci. Bull. 55, 2618-2624 (2010)

25. Pendry, J. B. \& Ramakrishna, S. A. Near-field lenses in two dimensions. J. Phys. Condens. Matter. 14, 8463-8479 (2002).

26. Salandrino, A. \& Engheta, N. Far-field subdiffraction optical microscopy using metamaterial crystals: theory and simulations. Phys. Rev. B 74, 075103 (2006).

27. Shvets, G., Trendafilov, S., Pendry, J. B. \& Sarychev, A. Guiding, focusing, and sensing on the subwavelength scale using metallic wire arrays. Phys. Rev. Lett. 99, 053903 (2007).

28. Ono, A., Kato, J. \& Kawata, S. Subwavelength optical imaging through a metallic nanorod array. Phys. Rev. Lett. 95, 267407 (2005).

29. Ikonen, P., Simovski, C., Tretyakov, S., Belov, P. \& Hao, Y. Magnification of subwavelength field distributions at microwave frequencies using a wire medium slab operating in the canalization regime. Appl. Phys. Lett. 91, 104102 (2007)

30. Zhao, Y. et al. Magnification of subwavelength field distributions using a tapered array of metallic wires with planar interfaces and an embedded dielectric phase compensator. New J. Phys. 12, 103045 (2010).

31. Han, S. et al. Ray optics at a deep-subwavelength scale: a transformation optics approach. Nano Lett. 8, 4243-4247 (2008).

32. Kildishev, A. V. \& Shalaev, V. M. Engineering space for light via transformation optics. Opt. Lett. 33, 43-45 (2008).

33. Tsang, M. \& Psaltis, D. Magnifying perfect lens and superlens design by coordinate transformation. Phys. Rev. B 77, 035122 (2008).

34. Li, J. S., Han, S., Zhang, S., Bartal, G. \& Zhang, X. Designing the Fourier space with transformation optics. Opt. Lett. 34, 3128-3130 (2009).

35. Kildishev, A. V. \& Narimanov, E. E. Impedance-matched hyperlens. Opt. Lett. 32, 3432-3434 (2007).

Proposal of an impedance-matched hyperlens for high transmission.

36. Smith, E. J., Liu, Z., Mei, Y. F. \& Schmidt, O. G. System investigation of a rolled-up metamaterial optical hyperlens structure. Appl. Phys. Lett. 95, 083104 (2009).
37. Wang, W. et al. Far-field imaging device: planar hyperlens with magnification using multi-layer metamaterial. Opt. Express 16, 21142-21148 (2008).

38. Lee, H., Liu, Z., Xiong, Y., Sun, C. \& Zhang, X. Development of optical hyperlens for imaging below the diffraction limit. Opt. Express 15, 15886-15891 (2007).

39. Xiong, Y., Liu, Z. W. \& Zhang, X. A simple design of flat hyperlens for lithography and imaging with half-pitch resolution down to $20 \mathrm{~nm}$. Appl. Phys Lett. 94, 203108 (2009)

40. Li, G. X., Tam, H. L., Wang, F. Y. \& Cheah, K. W. Half-cylindrical far field superlens with coupled Fabry-Perot cavities. J. Appl. Phys. 104, 096103 (2008)

41. Kerbst, J. et al. Enhanced transmission in rolled-up hyperlenses utilizing Fabry-Perot resonances. Appl. Phys. Lett. 99, 191905 (2011).

42. Schwaiger, S. et al. Rolled-up three-dimensional metamaterials with a tunable plasma frequency in the visible regime. Phys. Rev. Lett. 102, 163903 (2009).

43. Mei, Y. F. et al. Versatile approach for integrative and functionalized tubes by strain engineering of nanomembranes on polymers. Adv. Mater. 20, 4085-4090 (2008).

44. Gibbons, N., Baumberg, J. J., Bower, C. L., Kolle, M. \& Steiner, U. Scalable cylindrical metallodielectric metamaterials. Adv. Mater. 21, 3933-3936 (2009).

45. Yao, J. et al. Imaging visible light using anisotropic metamaterial slab lens. Opt. Express 17, 22380-22385 (2009).

46. Casse, B. D. F. et al. Super-resolution imaging using a three-dimensional metamaterials nanolens. Appl. Phys. Lett. 96, 023114 (2010).

47. Kawata, S., Ono, A. \& Verma, P. Subwavelength colour imaging with a metallic nanolens. Nat. Photonics 2, 438-442 (2008)

48. Qin, L. D., Park, S., Huang, L. \& Mirkin, C. A. On-wire lithography. Science 309, 113-115 (2005)

49. Aizpurua, J. et al. Optical properties of coupled metallic nanorods for field-enhanced spectroscopy. Phys. Rev. B 71, 235420 (2005).

50. Barnakov, Y. A. et al. Toward curvilinear metamaterials based on silver-filled alumina templates [Invited]. Opt. Mater. Express 1, 1061-1064 (2011).

51. Ma, C. B. \& Liu, Z. W. Designing super-resolution metalenses by the combination of metamaterials and nanoscale plasmonic waveguide couplers. J. Nanophotonics 5, 051604 (2011).

52. Sun, Z. \& Kim, H. K. Refractive transmission of light and beam shaping with metallic nano-optic lenses. Appl. Phys. Lett. 85, 642-644 (2004).

53. Verslegers, L. et al. Planar lenses based on nanoscale slit arrays in a metallic film. Nano Lett. 9, 235-238 (2009).

54. Parazzoli, C. G. et al. Performance of a negative index of refraction lens. Appl. Phys. Lett. 84, 3232-3234 (2004).

55. Pendry, J. B., Schurig, D. \& Smith, D. R. Controlling electromagnetic fields. Science 312, 1780-1782 (2006).

56. Shalaev, V. M. Transforming light. Science 322, 384-386 (2008).

57. Gómez-Reino, C., Pérez, M. \& Bao, C. Gradient-Index Optics: Fundamentals and Applications (Springer Verlag, 2002).

58. Verslegers, L., Catrysse, P. B., Yu, Z. F. \& Fan, S. Deep-subwavelength focusing and steering of light in an aperiodic metallic waveguide array. Phys. Rev. Lett. 103, 033902 (2009)

59. van Putten, E. G. et al. Scattering lens resolves sub-100 nm structures with visible light. Phys. Rev. Lett. 106, 193905 (2011).

60. Wu, Q., Feke, G. D., Grober, R. D. \& Ghislain, L. P. Realization of numerical aperture 2.0 using a gallium phosphide solid immersion lens. Appl. Phys. Lett. 75, 4064-4066 (1999).

61. Ippolito, S. B., Goldberg, B. B. \& Ünlü, M. S. High spatial resolution subsurface microscopy. Appl. Phys. Lett. 78, 4071-4073 (2001)

62. Levy, U. et al. Inhomogenous dielectric metamaterials with space-variant polarizability. Phys. Rev. Lett. 98, 243901 (2007).

63. Escobar, M. A., Berthome, M., Ma, C. B. \& Liu, Z. W. Focusing surface waves with an inhomogeneous metamaterial lens. Appl. Optics 49, A18-A22 (2010).

64. Hecht, E. Optics (Addison-Wesley, 2002).

65. Lerosey, G., De Rosny, J., Tourin, A. \& Fink, M. Focusing beyond the diffraction limit with far-field time reversal. Science 315, 1120-1122 (2007).

66. Lemoult, F., Lerosey, G., de Rosny, J. \& Fink, M. Resonant metalenses for breaking the diffraction barrier. Phys. Rev. Lett. 104, 203901 (2010).

Experimental demonstration of the resonant metalenses for far-field imaging in microwave frequencies based on time-reversal technique.

67. Sentenac, A., Chaumet, P. C. \& Belkebir, K. Beyond the rayleigh criterion: grating assisted far-field optical diffraction tomography. Phys. Rev. Lett. 97, 243901 (2006).

68. Maire, G. et al. Experimental demonstration of quantitative imaging beyond abbe's limit with optical diffraction tomography. Phys. Rev. Lett. 102, 213905 (2009).

69. Gazit, S., Szameit, A., Eldar, Y. C. \& Segev, M. Super-resolution and reconstruction of sparse sub-wavelength images. Opt. Express 17, 23920-23946 (2009).

70. Shechtman, Y., Eldar, Y. C., Szameit, A. \& Segev, M. Sparsity based sub-wavelength imaging with partially incoherent light via quadratic compressed sensing. Opt. Express 19, 14807-14822 (2011). 
71. Lerosey, G. et al. Time reversal of electromagnetic waves. Phys. Rev. Lett. 92, 193904 (2004).

72. Lemoult, F., Fink, M. \& Lerosey, G. Far-field sub-wavelength imaging and focusing using a wire medium based resonant metalens. Waves Random Complex Media 21, 614-627 (2011).

73. Lemoult, F., Fink, M. \& Lerosey, G. Revisiting the wire medium: an ideal resonant metalens. Waves Random Complex Media 21, 591-613 (2011).

74. Popoff, S. M. et al. Exploiting the time-reversal operator for adaptive optics, selective focusing, and scattering pattern analysis. Phys. Rev. Lett. 107, 263901 (2011).

75. Mosk, A. P., Lagendijk, A., Lerosey, G. \& Fink, M. Controlling waves in space and time for imaging and focusing in complex media. Nat. Photonics 6, 283-292 (2012).

76. Bartal, G., Lerosey, G. \& Zhang, X. Subwavelength dynamic focusing in plasmonic nanostructures using time reversal. Phys. Rev. B 79, 201103 (2009).

77. Lemoult, F., Fink, M. \& Lerosey, G. A polychromatic approach to far-field superlensing at visible wavelengths. Nat. Commun. 3, 889 (2012).

78. Turpin, T. M., Gesell, L. H., Lapides, J. \& Price, C. H. Theory of the synthetic aperture microscope. Proc. SPIE 2566, 230-240 (1995).

79. Woodford, P., Turpin, T. M., Rubin, M. W., Lapides, J. \& Price, C. H. The synthetic aperture microscope: experimental results. Proc. SPIE 2751, 230-240 (1996).

80. Lauer, V. New approach to optical diffraction tomography yielding a vector equation of diffraction tomography and a novel tomographic microscope. J. Microsc. 205, 165-176 (2002).

81. Alexandrov, S. A., Hillman, T. R., Gutzler, T. \& Sampson, D. D. Synthetic aperture Fourier holographic optical microscopy. Phys. Rev. Lett. 97, 168102 (2006).

82. Belkebir, K., Chaumet, P. C. \& Sentenac, A. Superresolution in total internal reflection tomography. J. Opt. Soc. Am. A 22, 1889-1897 (2005).

83. Girard, J. et al. Nanometric resolution using far-field optical tomographic microscopy in the multiple scattering regime. Phys. Rev. A 82, 061801(R) (2010).

84. Guenneau, S., Movchan, A., Petursson, G. \& Ramakrishna, S. A. Acoustic metamaterials for sound focusing and confinement. New J. Phys. 9, 399 (2007).

85. Ao, X. Y. \& Chan, C. T. Far-field image magnification for acoustic waves using anisotropic acoustic metamaterials. Phys. Rev. E 77, 025601(R) (2008).

86. Lee, H. J., Kim, H. W. \& Kim, Y. Y. Far-field subwavelength imaging for ultrasonic elastic waves in a plate using an elastic hyperlens. Appl. Phys. Lett 98, 241912 (2011).

87. Li, J. S., Fok, L., Yin, X. B., Bartal, G. \& Zhang, X. Experimental demonstration of an acoustic magnifying hyperlens. Nat. Materials 8, 931-934 (2009). Experimental demonstration of a $2 \mathrm{D}$ acoustic hyperlens for deepsubwavelength imaging over a broadband frequency range with low loss.
88. Torrent, D. \& Sanchez-Dehesa, J. Anisotropic mass density by two-dimensional acoustic metamaterials. New J. Phys. 10, 023004 (2008).

89. Zhu, J. et al. A holey-structured metamaterial for acoustic deep-subwavelength imaging. Nat. Phys. 7, 52-55 (2011).

90. Christensen, J., Fernandez-Dominguez, A. I., De Leon-Perez, F., MartinMoreno, L. \& Garcia-Vidal, F. J. Collimation of sound assisted by acoustic surface waves. Nat. Phys. 3, 851-852 (2007).

91. Zhang, S., Xia, C. \& Fang, N. Broadband acoustic cloak for ultrasound waves Phys. Rev. Lett. 106, 024301 (2011)

92. Mei, J. et al. Dark acoustic metamaterials as super absorbers for low-frequency sound. Nat. Commun. 3, 756 (2012).

93. Lu, M.-H. et al. Extraordinary acoustic transmission through a $1 \mathrm{D}$ grating with very narrow apertures. Phys. Rev. Lett. 99, 174301 (2007).

94. Lemoult, F., Fink, M. \& Lerosey, G. Acoustic resonators for far-field control of sound on a subwavelength scale. Phys. Rev. Lett. 107, 064301 (2011)

95. de Rosny, J. \& Fink, M. Overcoming the diffraction limit in wave physics using a time-reversal mirror and a novel acoustic sink. Phys. Rev. Lett. 89, 124301 (2002).

96. Xiao, S. M. et al. Loss-free and active optical negative-index metamaterials. Nature 466, 735-738 (2010).

97. Boltasseva, A. \& Atwater, H. A. Low-loss plasmonic metamaterials. Science 331, 290-291 (2011)

98. Naik, G. V., Liu, J., Kildishev, A. V., Shalaev, V. M. \& Boltasseva, A. Demonstration of $\mathrm{Al}: \mathrm{ZnO}$ as a plasmonic component for near-infrared metamaterials. Proc. Natl Acad. Sci. USA 109, 8834-8838 (2012).

99. Hu, H., Ma, C. \& Liu, Z. Plasmonic dark field microscopy. Appl. Phys. Lett. 96, 113107 (2010)

\section{Acknowledgements}

We acknowledge financial support from NSF-ECCS under Grant No. 0969405 and NSFCMMI under Grant No. 1120795. We also thank Joshua Hoemke for the manuscript revisions, and Geoffroy Lerosey, Fabrice Lemoult and Jensen Li for their discussions on figures.

\section{Additional information}

Competing financial interests: The authors declare no competing financial interests

Reprints and permission information is available online at http://npg.nature.com/ reprintsandpermissions/

How to cite this article: Lu, D. et al. Hyperlenses and metalenses for far-field super-resolution imaging. Nat. Commun. 3:1205 doi: 10.1038/ncomms2176 (2012) 